\title{
Resistive Micromegas for sampling calorimetry
}

\author{
C. Adloff, M. Chefdeville*, A. Dalmaz, C. Drancourt, R. Gaglione, N. Geffroy, J. \\ Jacquemier, Y. Karyotakis, I. Koletsou, F. Peltier, J. Samarati, G. Vouters \\ LAPP, Laboratoire d'Annecy-le-Vieux de Physique des Particules, Université de Savoie, \\ CNRS/IN2P3, 9 Chemin de Bellevue - BP 110, 74941 Annecy-le-Vieux Cedex, France \\ E-mail: chefdevi@lapp.in2p3. fr
}

\begin{abstract}
Micromegas is an attractive option for a gaseous sampling calorimeter. It delivers proportional and fast signals, achieves high efficiency to minimum ionising particles with a compact design and shows well-uniform performance over meter-square areas. The current R\&D focuses on largesize spark-protected Micromegas with integrated front-end electronics. It targets an application at future linear colliders (LC) and possible upgrades of LHC experiments for the running at high luminosity. In the later case, occasional sparking should be suppressed to avoid dead-time and technical solutions using resistive coatings are investigated. Small prototypes of resistive and non-resistive Micromegas were constructed and tested in a beam at DESY. Results are reported with emphasis on the impact of the resistive layer on the detector performance.
\end{abstract}

Technology and Instrumentation in Particle Physics 2014,

2-6 June, 2014

Amsterdam, the Netherlands

\footnotetext{
* Speaker.
} 


\section{Introduction}

Micromegas is one detector option for a digital hadron calorimeter at a future linear collider. Large-area prototypes of $1 \times 1 \mathrm{~m}^{2}$ [1] achieve excellent performance for the targeted application [2]. For higher rate applications, however, occasional sparking creates prohibitive dead-time and should therefore be suppressed. Spark suppression was demonstrated in resistive Micromegas [3]. The principle is to introduce a grounded resistive electrode which capacitively couples to the readout electrode through an insulating layer. At high detector current, the potential of the resistive layer changes significantly, reducing the amplification field and quenching the spark at an early development stage. The use of resistive Micromegas as a sampling element in a calorimeter, however, is not straight-forward. High currents are generated in high-energy showers or when operating at high rates. Also, the variations of the electrical potential over the resistive layer surface during an avalanche result in a broader pad response function. The geometrical and electrical properties of the films should be optimised. A first study was conducted with small prototypes.

\section{Experimental set-up}

Prototypes Non-resistive Micromegas prototypes are small replica of the large-area prototypes, consisting of a Printed Circuit Board (PCB) with a $16 \times 16$ matrix of $2561 \times 1 \mathrm{~cm}^{2}$ pads, 4 front-end ASICs, a Bulk Micromegas and flat connectors. A $3 \mathrm{~mm}$ drift gap is defined by a drift electrode glued on a square plastic frame that also serves for gas distribution. Readout is done with the acquisition system of the large-area prototypes described in [1]. Resistive prototypes integrate resistive elements and insulators between the anode plane and the Bulk mesh. Two designs were studied with the goal to limit cross-talk (Fig. 1).
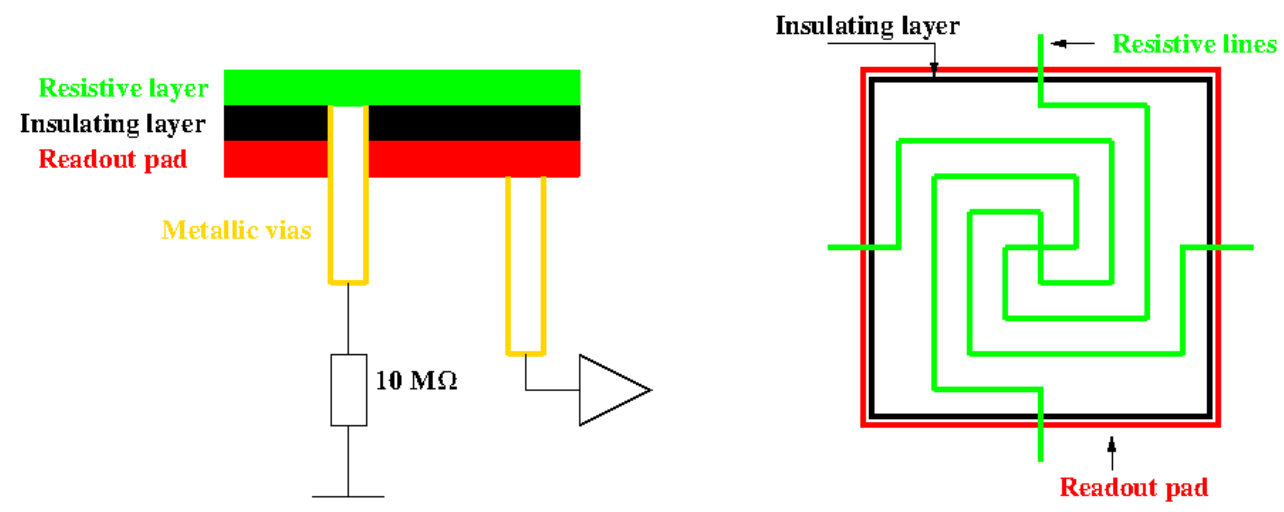

Figure 1: Not-to-scale drawings of the two resistive configurations using pads (left) and strips (right).

The first consists of a $64 \mu \mathrm{m}$ thin insulating sheet laminated on the anode PCB, on top of which a resistive paste of a few $\mathrm{M} \Omega / \mathrm{sq}$ is deposited. The resistive paste is segmented into $1 \times 1 \mathrm{~cm}^{2}$ pads which are connected to ground through the PCB by metallic vias (1 per pad). The second design is based on a thin insulating sheet, coated with a resistive paste patterned into strips. The pattern consists of windings of strips ( $100 \mu \mathrm{m}$ width, $50 \mu \mathrm{m}$ inter-strip gap, 1 winding per pad). Windings are inter-connected (4 connections per pad) and grounded to one PCB side. After an electron 
avalanche, the charge density spreads along the strip surface. Thanks to the winding pattern, the charge distribution is already quite broad when reaching the neighboring pads where it remains below threshold. Compared to a non-segmented resistive layer, this configuration is expected to show much reduced cross-talk.

Testbeam Three standard and two resistive prototypes were constructed and mounted on a mechanical structure. The detector stack was installed in the beam line T22 at DESY and exposed to $1-5 \mathrm{GeV}$ electrons. Standard prototypes are used as a telescope and were placed at position 1-3-5 along the beam direction. Resistive prototypes were inserted at position 2 and 4. A gas mixture of $\mathrm{Ar} / \mathrm{CO}_{2}$ 90/10 was flushed in the prototypes, connected in series. Triggering equipments are 3 scintillator paddles of $6 \times 16 \mathrm{~cm}^{2}$ readout by photomultiplier tubes: 2 upstream of the structure, 1 downstream. To trigger on electrons traversing the whole stack, signals from the 3 tubes are used. By placing steel absorbers in front of the stack, beam electrons are showering. In this case, the trigger uses signals from the 2 upstream tubes. ASICs feature 3 discriminators with tunable thresholds and a memory which is read out upon reception of the trigger signal. Also, the readout board of each prototype is equipped with a 12-bit ADC to digitise the shaper output of all ASIC channels.

\section{Response to traversing electrons and sparks}

The response of resistive prototypes was studied at various mesh voltages. The scan is performed with a $3 \mathrm{GeV}$ electron beam focused to a $2 \times 2 \mathrm{~cm}^{2}$ spot. The beam is delivered at a continuous rate of $1 \mathrm{kHz}$ such that rate-induced gain losses are negligible (cf. section 5). The first and last prototypes are used as a telescope and their voltages are fixed. The magnitude of the signal (i.e. Landau distribution) is measured with the readout board ADCs. Then, the lateral spread of signals on the pad matrix is investigated based on the number of hits recorded by the ASICs. About $5 \times 10^{4}$ events are recorded at each voltage. A second scan is finally performed at the highest rate on-site $(\sim 200 \mathrm{kHz})$ to verify spark suppression by monitoring prototype voltages and currents.
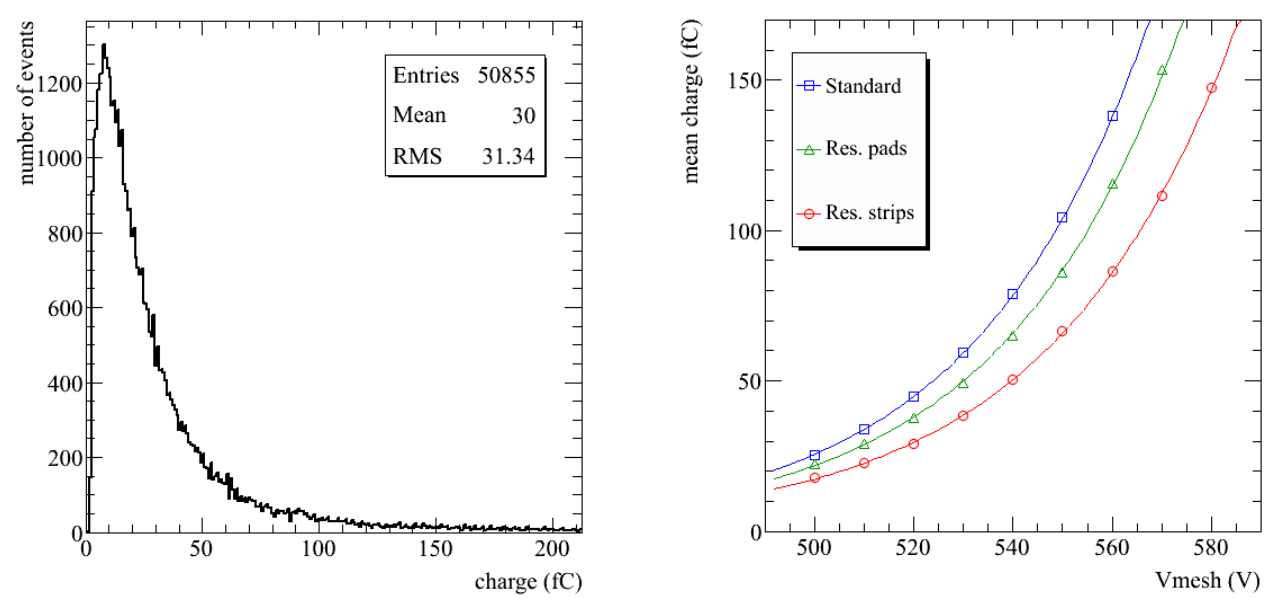

Figure 2: (left) Charge distribution. (right) Mean charge versus mesh voltage in 3 prototypes. 
Charge distribution Analogue (ADC counts) and digital (hit) information is used to construct the Landau distribution. The charge per event is calculated as a sum of pedestal-subtracted ADC values from pads with a hit. Using the known calibration constant of the electronics chain, the sum is then converted into charge. The Landau distribution obtained with a resistive prototype is shown in Fig. 2 (left). The exponential gain-to-voltage relation is verified in Fig. 2 (right). Similar slopes are measured in the 3 prototypes $(0.027-0.028 / \mathrm{V})$ but absolute values are different. Compared with the standard prototype, $10 \mathrm{~V}$ and $20 \mathrm{~V}$ more are necessary to measure the same charge in the resistive prototypes (pad and strip respectively). At $510 \mathrm{~V}$, the charge ratio is 0.85 and 0.7 which partly results from the capacitive coupling. The smaller ratio (strip design) could be explained by a lower field above the inter-strip regions and a weaker coupling due to the absence of a metallic via.

Number of hit distribution In a digital calorimeter, only the number of pad signals above threshold are measured (so-called hits). The number of hits depends on the track angle and intersect with the pad plane. With a beam spot of $2 \times 2 \mathrm{~cm}^{2}$, the second dependence is washed out. The track angle is constrained close to $90^{\circ}$ by selecting events with single aligned hits in the first and last telescope prototypes. A search region of $3 \times 3$ pads is chosen around the expected track position in the tested prototypes. These two cuts reduce the contamination from bremsstrahlung photons and electrons showering in the prototype material.
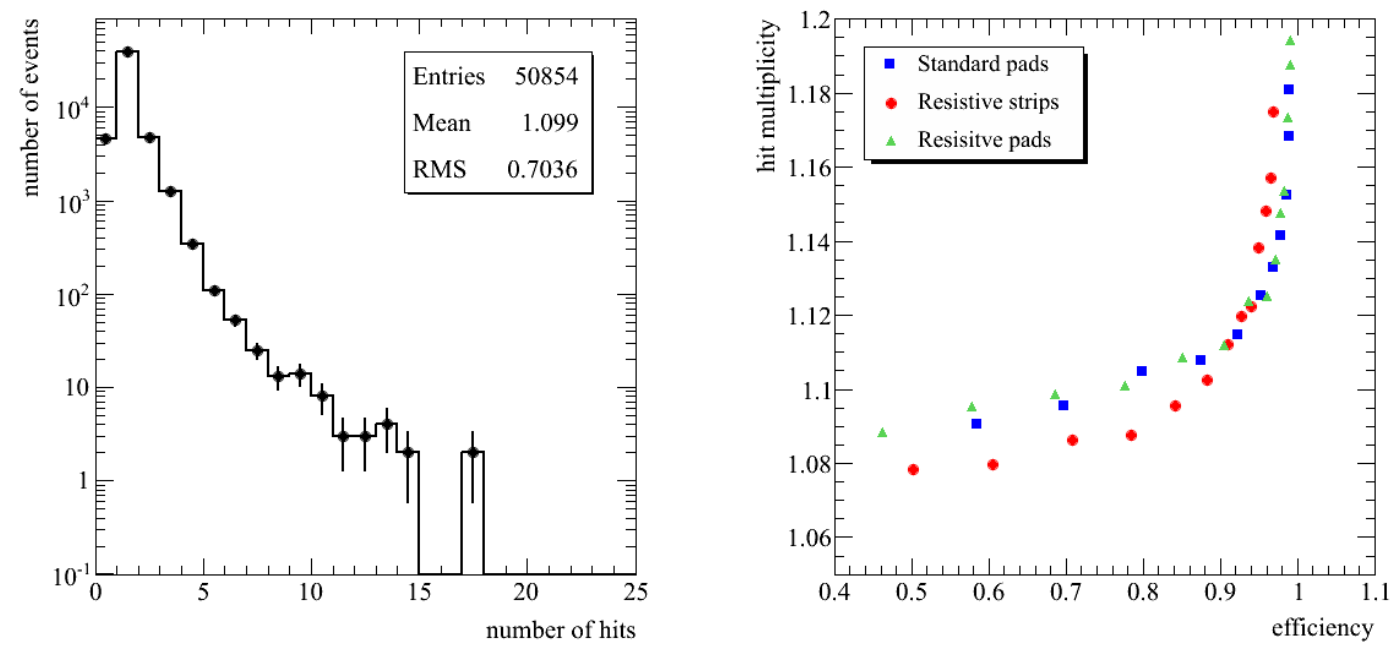

Figure 3: (left) Number of hit distribution.(right) Hit multiplicity versus efficiency in 3 prototypes.

The raw distribution of number of hits in the resistive strip prototype is shown in Fig. 3 (left). It has a mean of 1.1 and yields a hit multiplicity of 1.7 (defined as the mean excluding the zero-hit bin). After applying cuts, the multiplicity drops to 1.1 which is the nominal value obtained with large-area prototypes [2]. The measured performance are summarised in Fig. 3 (right) where the hit multiplicity is plotted versus efficiency. All prototypes achieve an efficiency larger than $95 \%$. Hit multiplicities agree well at high efficiency. The resistive layers have a negligible impact on the pad response function, thanks to the adopted designs. Interestingly, the efficiency of the strip design saturates a few percent below that of the other designs. Probably because the active area of this prototype is reduced by the inter-strip regions of lower field. 
Spark suppression The voltage-current history of the standard prototype during the second voltage scan at $\sim 200 \mathrm{kHz}$ is shown in Fig. 4 (left). Large current spikes occur more frequently as voltage is increased and are therefore likely caused by sparking. The resulting voltage drops are also shown in the figure. From the known beam rate, the spark probability per traversing electron is easily calculated. At $510 \mathrm{~V}$ ( $\sim 95 \%$ efficiency), it equals $\sim 10^{-7}$ per traversing electron. On the contrary, the 2 resistive prototypes are very quite. Few spikes corresponding to the ramped-up current are visible in Fig. 4 (right). Accordingly, the resistive designs fully suppress sparking.

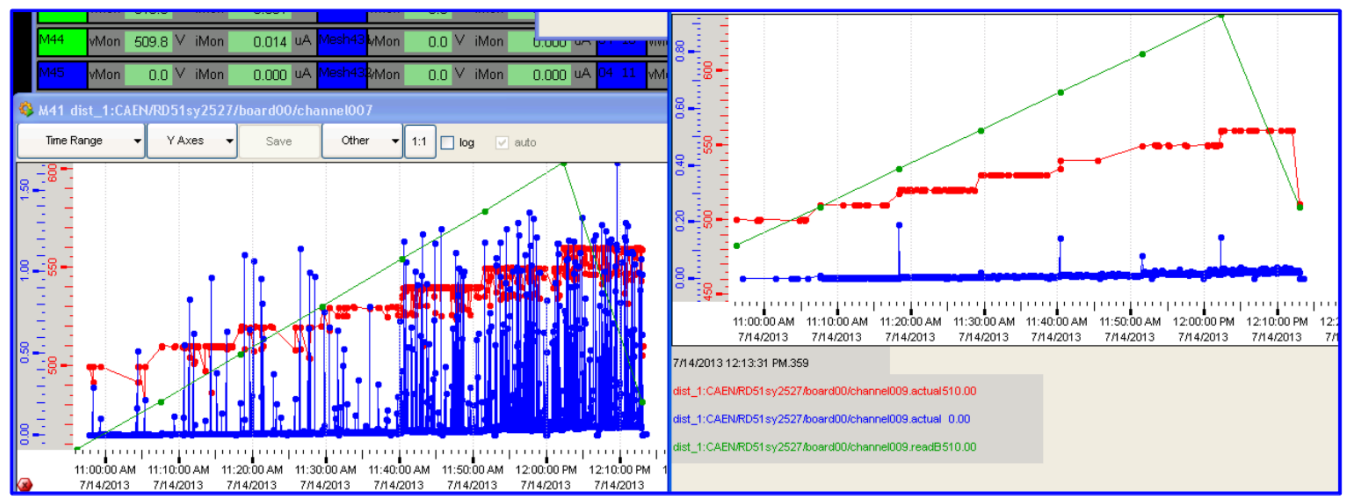

Figure 4: Screen capture of the slow-control application showing mesh current (in blue) and voltage (in red) history of standard (left) and resistive (right) prototypes during the voltage scan $(500-560 \mathrm{~V})$.

\section{Signal linearity in electron showers}

Indirect measurement The linearity of a sampling calorimeter to electrons can be indirectly measured with a single layer. At a given electron energy, the layer response (charge distribution or number of hit distribution) is measured for various numbers of steel plates placed upstream of the layer (Fig. 5 (left)). The longitudinal profile of the shower is given by the mean signal versus steel thickness (Fig. 5 (right)). The total signal that would be measured in a sampling calorimeter is then proportional to the integral of the profile. The later is best estimated by fitting the profile and calculating the integral of the fit function. Repeating this exercise at various energies, the calorimeter response can be indirectly measured and its linearity checked.

Results with charge readout The analogue response to electrons of a virtual calorimeter over the $1-5 \mathrm{GeV}$ energy range is shown in Fig. 6 (left), for the 3 upstream prototypes. It decreases with the position along the beam direction $(O z)$ as a result of the increased material (chamber mechanics and PCB, air gaps) between the front of the stack and the prototype. The response of the resistive strip prototype (at $z=2$ ) seems linear up to $4 \mathrm{GeV}$, like the response of the 2 standard prototypes (at $z=1$ and $z=3$ ). Deviations from linearity observed at $5 \mathrm{GeV}$ are likely due to a poor fit of the profile resulting in an under-estimated integral of the fit function (Fig. 5 (right)). This deviation is observed for the 3 prototypes which suggests that it is not caused by the resistive layer. Most probably, linearity in resistive prototypes is preserved over the tested energy range but might be spoiled at higher energy. 

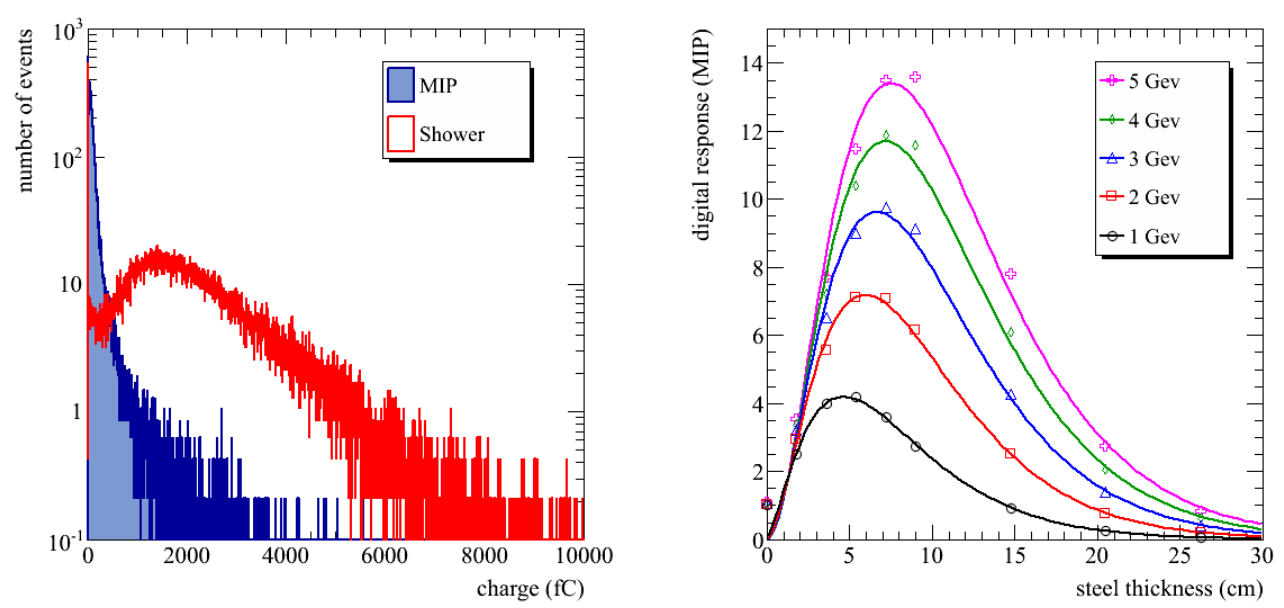

Figure 5: (left) Charge distribution from $5 \mathrm{GeV}$ electron with (red) and without (blue) absorbers upstream of the sampling prototype. (right) Mean charge versus steel thickness measured at various electron energies.


Figure 6: (left) Analogue response of a virtual calorimeter equipped with resistive $(z=2)$ and standard Micromegas $(z=1$ and $z=3$ ). (right) Digital response for a pad threshold of $\sim 1-2 \mathrm{fC}$.

Results with digital readout The analysis was performed on the digital data ( $\sim 1-2 \mathrm{fC}$ threshold). The digital response plotted in Fig. 6 (right) shows a strong saturation for the 3 prototypes. With a pad size of $1 \times 1 \mathrm{~cm}^{2}$ and a Molière radius of $\sim 2 \mathrm{~cm}$, the lateral sampling of the shower is poor which explains the geometrical saturation. A Micromegas electromagnetic calorimeter should therefore have smaller pads or measure charge. The situation is somewhat similar for hadron calorimetry, though less problematic because of the wider lateral spread of hadron showers. 


\section{Rate-induced effects}

Efficiency loss The beam rate was progressively increased from $0.5 \mathrm{kHz}$ up to $\sim 200 \mathrm{kHz}$ by opening the collimators along the beam line (with a beam spot size of $2 \times 2 \mathrm{~cm}^{2}$ at the prototypes). Using the first and last standard prototypes as a telescope, the efficiency of the other prototypes was measured for 3 values of thresholds. The scan proceeded in 7 runs of 10 minutes. For each run, the variations of efficiency with time were determined. The mean efficiency during a run is normalised to the mean efficiency during the first run (close to $95 \%$ for both resistive prototypes) when rate-effects were negligible. This normalisation allows easier comparison of data recorded at different thresholds. The normalised efficiency variations with rate are plotted in Fig. 7 (left) for the resistive strip prototype.

Rate effects become significant above $40 \mathrm{kHz}$, i.e. above $10 \mathrm{kHz} / \mathrm{cm}^{2}$. The efficiency loss is more important at higher thresholds as expected from the shape of the Landau distribution. Similar trends are observed for the resistive pad prototype. Neglecting sparking at high rates, the standard prototype efficiency is essentially constant during the scan.
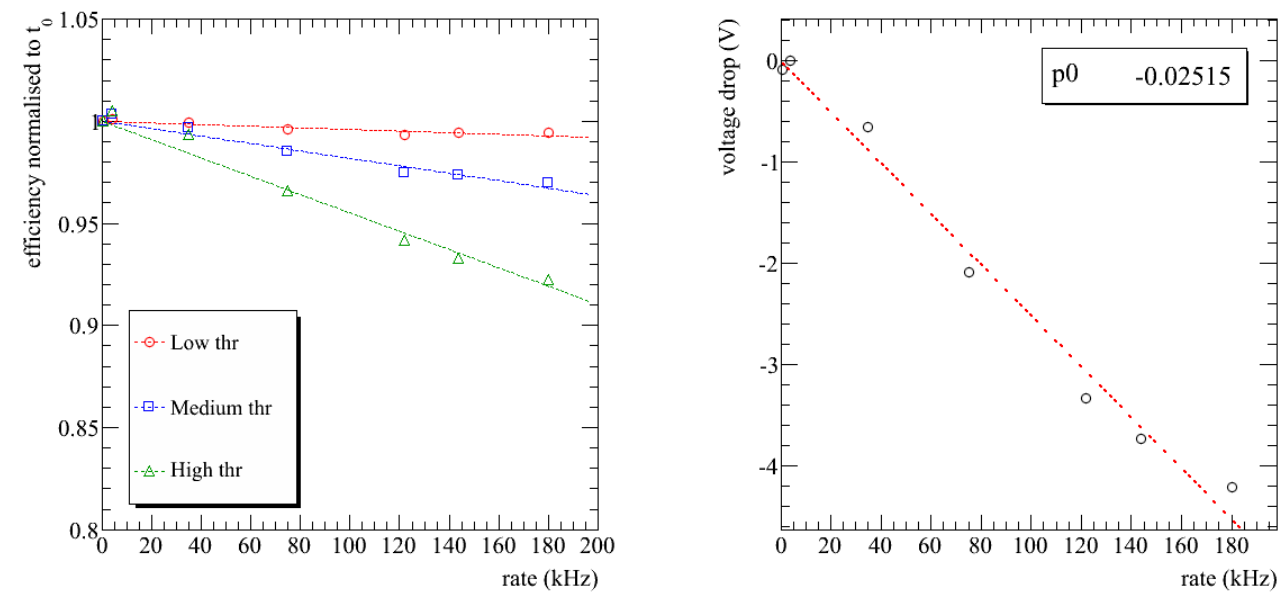

Figure 7: (left) Efficiency versus rate measured in the resistive strip prototype at 3 values of threshold. (right) Voltage drop necessary to account for the measured efficiency loss as a function of rate.

Voltage drop The analogue readout was too slow for being efficiently used during the rate scan but valuable information was extracted from the digital data alone. Using information from Fig. 2, the data can be used to estimate the voltage drop across the amplification gap as a function of rate. As shown in Fig. 7 (right), the relation is approximately linear as expected from the Ohmic law and from the fact that over the tested rate range, mesh current and rate are roughly proportional. The effective resistance of the prototype can be deduced from a linear fit to the data points. Assuming 30 primary electrons per traversing beam particle and a gas gain of $2 \times 10^{3}$, a resistance of $\sim 1 \mathrm{G} \Omega$ is found. Considering that the sheet resistance is of a few MOhm/sq and the strip width is $100 \mu \mathrm{m}$, this value is expected if an effective strip length of a few $\mathrm{cm}$ is assumed. 


\subsection{Conclusions}

For the first time, the use of resistive Micromegas for calorimetry is investigated. Two different resistive prototypes of small size were constructed and tested in beam at DESY. Compared to non-resistive prototypes, sparking is fully suppressed. Also, a smaller charge was measured at the readout pads which is compensated by applying 10-20 V more across the amplification gap. Thanks to a careful design, lateral spread of the avalanche charge onto the anode plane is avoided. Signal linearity in electron showers is probably not affected up to $5 \mathrm{GeV}$, highest energy on-site. The two resistive prototypes show a reduced rate capability with a gas gain loss of a few percents at $10 \mathrm{kHz} / \mathrm{cm}^{2}$. This capability will be improved by lowering the coating resistivity of future prototypes.

Acknowledgements The authors wish to thank the French Agence Nationale de la Recherche (ANR) for financial support and the DESY research centre for providing the gas mixture, steel absorbers and technical guidance during the testbeam.

\section{References}

[1] C. Adloff et al., Construction and test of a $1 \times 1 \mathrm{~m}^{2}$ Micromegas chamber for sampling hadron calorimetry at future lepton colliders, Nucl. Instr. and Meth. A 729 (2013) 90.

[2] C. Adloff et al., Test in a beam of large-area Micromegas chambers for sampling calorimetry, To appear in Nucl. Instr. and Meth. A.

[3] T. Alexopoulos et al., A spark-resistant bulk-micromegas chamber for high-rate applications, Nucl. Instr. and Meth. A 640 (2011) 110. 